\title{
CURRICULUM VITAE
}

\section{XINYU LIU}

Department of Physics, University of Notre Dame

225 Nieuwland Science Hall, Notre Dame, IN 46556

Phone: (574)631-9787 (o); (574) 855-7737 (cell) Fax: (574)631-5952Ｅ-mail: xliu2@,nd.edu

\section{PROFESSIONAL APPOINTMENTS}

University of Notre Dame Research Assistant Professor of Physics 2004-present

Conducting, among others, research on spin-based processes in semiconductors and their nanostructures funded by NSF, DOE, ONR, and DARPA. Providing leadership in research projects and supervising MS and Ph.D. graduate students.

University of Notre Dame $\quad$ Postdoctoral Research Associate 2002-2004

Played a major part in one of the national top priority research projects - the SPINS program funded by DARPA. Conducted physical studies of spin-based processes in magnetic semiconductors.

University of Notre Dame $\quad$ Graduate Research Assistant 2000-2002

Developed ferromagnetic semiconductor materials and carried out magnetic, electronic, optical, and magnetic resonance studies on these materials.

\section{University of Notre Dame Teaching Assistant}

1996-2000

The principal duties were to serve as assistant to a faculty instructor, as homework or exam grader, tutorial leader or laboratory instructor, and as occasional lecturer in undergraduate or graduate courses. Achieved Department Teaching Assistant Award in 2000.

\section{U. of Sci. \& Tech. of China Software Programmer (part-time) 1994-1996}

Developed and programmed computer management information systems in various companies as part-time software programmer.

\section{EDUCATION}

University of Notre Dame Ph.D. Condensed Matter Physics

$01 / 2003$

Dissertation: Thin Semiconductor Alloy Films: Fabrication and Physical Properties

Directed by Professor Jacek K. Furdyna

U. of Sci. \& Tech. of China M.S. Physics

07/1996

Thesis: Zhejiang Three Lions Cement Co., Ltd Network Management Information System

Directed by Professor Pingsong Hong
U. of Sci. \& Tech. of China
B. Eng. Applied Physics
$07 / 1993$

\section{RECENT/CURRENT RESEARCH}

Research Activities and Interests:

- Spin phenomena in semiconductor nanostructures for spintronic applications;

- Developing, fabricating, and characterizing nanostructures based on magnetic semiconductors, and hybrid systems such as semiconductor/superconductor and semiconductor/ferromagnet;

- Studies of low-dimensional semiconductor structures (quantum wells, quantum wire, quantum dots, coupled quantum dots, etc.) grown by MBE;

- Semiconductor optoelectronic devices and materials, including semiconductor lasers, photodetectors, solar cells, and their integration for various applications.

- Materials science researches using ferromagnetic resonance, electronic and spin transport, neutron scattering, magneto-optical imaging, ultrafast pump-probe spectroscopy, E-beam lithograph, AFM, SEM, TEM, channeling Rutherford backscattering (c-RBS), and channeling particle induced x-ray emission (c-PIXE). 
Synergistic Activities:

Collaborating extensively with over 30 other research institutions - including Universities, National and Government Laboratories, and Research Institutes in the US and abroad - both by providing research specimens and by sharing important research findings with collaborators in those institutions. Feedback from these outside collaborations not only improves the quality of the materials fabricated at Notre Dame, but also extends the research in the directions of new device design.

Program Committee Member for Electronic Materials Conference 2008-2010

\section{RESEARCH HIGHLIGHTS}

- 205 publications in top peer-reviewed journals, such as Nature Physics (1), Nature Materials (2), Nano Letters (1), Physical Review Letters (11), Applied Physics Letters (37), and Physical Review B (37); Over 2700 citations on articles published; Total citing articles 1255 (without self-citations); with an h-index of 26 as of Apr. 2011.

- Development of ultra-high efficiency multijunction solar cell using lattice-matched II/VI $(\mathrm{ZnCdMg})(\mathrm{SeTe})$ and $\mathrm{III} / \mathrm{V}(\mathrm{AlGa})(\mathrm{AsSb})$ direct bandgap materials grown on $\mathrm{GaSb}$ and InAs substrates.

- Development of molecular beam epitaxy (MBE) growth technology for fabrication of III-Mn-V ferromagnetic semiconductors (mixed alloys and digital alloys): GaMnAs, InMnAs, GaMnSb and InMnSb

- Comprehensive magnetic anisotropy studies in III-Mn-V alloys via ferromagnetic resonance (FMR). Identification of uniaxial and cubic anisotropy in GaMnAs thin films. This information is of key importance for operation of spin-injection-based spintronic devices

- Studies of the effect of low temperature annealing on electrical and magnetic properties of GaMnAs, showing that the increase of the ferromagnetic transition temperature indirectly linked to the rearrangement of $\mathrm{Mn}$ atoms in the crystal lattice. Identification of the thermodynamic limit of free hole concentration, and hence of the mechanism which limits the ferromagnetic transition temperature

- Studies of the effect of Be co-doping on magnetic properties of ferromagnetic GaMnAs, and identification of the mechanism which (counter-intuitively) leads to the observed decrease of the transition temperature caused by such co-doping

- Comprehensive investigation of the index of refraction for II-VI-based semiconductor compounds, including modeling of optical dispersion in these materials

\section{PRESENTATIONS}

"II-VI Heterostructures Obtained by Encapsulation of Colloidal CdSe Nanowires by MBE Deposition of ZnSe", Xinyu Liu, A. M. Mintairov, J. Herzog, F. Vietmeyer, R. Pimpinella, M. Kuno, J. L. Merz, T. H. Kosel, M. Dobrowolska, and J. K. Furdyna, $27^{\text {th }}$ North American Molecular Beam Epitaxy Conference - oral presentation (2010).

"MBE growth of lattice-matched 6.1Å II-VI on GaSb substrates", X. Liu, D. Ding, S. Wang, S.-N. Wu, X. Zhang, J. Fan, J.-J. Li, X. Lu, S. R. Johnson, D. J. Smith, J. K. Furdyna, and Y.-H. Zhang, The 2010 Electronic Materials Conference - oral presentation (2010).

"Magnetic Anisotropy, Interlayer Exchange Coupling, and Spin Manipulation in GaMnAs", X. Liu, Physics Seminar, Miami University - oral presentation (Invited) (2.17.2010).

"Spin dynamics and manipulation in GaMnAs alloys", X. Liu, Y. Y. Zhou, E. Harley, L. E. McNeil, J. Wang, J. Qi, Y. Xu, A. Steigerwald, N. Tolk, J. P. Zahn, A. Gamouras, S. March, K. C. Hall, J. K. Furdyna, 2010 SPIE Photonics West - oral presentation (Invited) (2010).

"Origin of magnetic circular dichroism in GaMnAs: giant Zeeman splitting vs. spin dependent density of states", M. Berciu, R. Chakarvorty, Y. Y. Zhou, M. T. Alam, K. Traudt, R. Jakiela, A. Barcz, T. Wojtowicz, X. Liu, J. K. Furdyna, and M. Dobrowolska, The 2009 Electronic Materials Conference - oral presentation (2009).

"Temperature Dependence of Anomalous Hall Effect in Metallic (Ga,Mn)As films", X. Liu, Z. Ge, S. Shen, M. Dobrowolska and J. K. Furdyna, 2009 APS March meeting - oral presentation (2009).

"Scaling of Anomalous Hall Effect in Metallic (Ga,Mn)As films", X. Liu, Z. Ge, S. Shen, M. Dobrowolska and J. K. Furdyna, The Fest-Symposium "Magnetic excitations in Semiconductors - Bridges to the Next Decade" - poster presentation (2008). 
"Ferromagnetic semiconductor memories: devices beyond binary logic", X. Liu, J.K. Furdyna, M. Dobrowolska, S. Lee, L. P. Rokhinson, Notre Dame MIND - poster presentation (2008).

"Magnetic Resonance and Spin-Wave Excitations in Ferromagnetic Semiconductor GaMnAs", X. Liu, Condensed Matter Seminar, Department of Physics, University of Notre Dame - oral presentation (Invited) (11.16.2007).

"Ferromagnetic Resonance Study in Ferromagnetic Semiconductors: Effects of Magnetic Anisotropy", X. Liu, 53rd Midwest Solid State Physics Conference - oral presentation (Invited) (2006).

"Temperature-dependent photoluminescence of vertically stacked self-assembled CdSe quantum dots in ZnSe", X. Liu, M. Dobrowolska, J. K. Furdyna, and S.Lee, The 12th International Conference on Modulated Semiconductor Structures (MSS 12) - poster presentation (2005).

"Magnetic anisotropy of strain-engineered InMnAs ferromagnetic films and easy-axis manipulation from out-ofplane to in-plane orientations", X. Liu, W. L. Lim, Z. Ge, S. Shen, T. Wojtowicz, K. M. Yu, W. Walukiewicz, M. Dobrowolska, and J. K. Furdyna, 27th Int. Conf. on Physics of Semiconductors - oral presentation (2004).

"Ferromagnetic resonance in modulation-doped GaMnAs/GaAlAs:Be heterostructures", X. Liu, W. L. Lim, M. Dobrowolska, J. K. Furdyna, and T. Wojtowicz, 2004 APS March meeting - oral presentation (2004).

"External control of the direction of magnetization in ferromagnetic InMnAs/GaSb heterostructures", X. Liu, W. L. Lim, L. V. Titova, T. Wojtowicz, M. Kutrowski, K. J. Yee, M. Dobrowolska, J. K. Furdyna, S. J. Potashnik, M. B. Stone, P. Schiffer, I. Vurgaftman, and J. R. Meyer, 11-th International Conference on Narrow Gap Semiconductors (NGS-11)- poster presentation (2003).

"Ferromagnetic resonance in GaMnAs: effects of magnetic anisotropy", X. Liu, Y. Sasaki, and J. K. Furdyna, 2003 APS March meeting - oral presentation (2003).

"Sinusoidally-Modulated III-V Semiconductor Superlattices by Molecular Beam Epitaxy", X. Liu, Y. Sasaki, P. M. Reimer, S. Lee, J. K. Furdyna, The 2001 Electronic Materials Conference - oral presentation (2001).

"Modification of Magnetic Properties of GaMnAs by Paramagnetic $\mathrm{Zn}_{1-\mathrm{x}} \mathrm{Mn}_{\mathrm{x}} \mathrm{Se}$ Overlayers", Xinyu Liu, Yuji Sasaki, and Jacek Furdyna, 2001 APS March meeting - oral presentation (2001).

\section{GRANTS AND SPONSORED PROGRAMS}

Title: Electron Spin Effects in Semiconductor Nanostructures

Agency: NSF/DMR/CMP

Total Award Amount: \$600,000, Total Award Period Covered: 09/01/10 - 08/31/14.

PI: Prof. M. Dobrowolska; Co-PI: Prof. J. K. Furdyna and Prof. X. Liu.

Title: Collaborative Proposal: Novel Multijunction Solar Cells for Space and Terrestrial Applications Agency: NSF/ECCS

Total Award Amount: \$139,999, Total Award Period Covered: 07/01/10 - 06/30/13.

PI: Prof. Y.-H. Zhang; Co-PI: Prof. J. K. Furdyna and Prof. X. Liu.

Title: Electron Spin Effects in Semiconductor Nanostructures

Agency: NSF/DMR/CMP

Total Award Amount: \$480,000, Total Award Period Covered: 08/01/06 - 07/31/11.

PI: Prof. M. Dobrowolska; Co-PI: Prof. J. K. Furdyna and Prof. X. Liu.

Title: Spin manipulation of confined states

Agency: Civil Research and Development Foundation (CRDF)

Total Award Amount: \$12,600, Total Award Period Covered: 03/15/08 - 03/14/10

PI: Prof. J. K. Furdyna; Co-PI: Prof. M. Dobrowolska and Prof. X. Liu.

Title: Design, Fabrication and Testing of Thin-Layer III-Mn-V Ferromagnetic Semiconductor Structures for Tunable Wavelength Photo-Detectors

Agency: NSA (as Subcontact from Laboratory for Physical Sciences)

Total Award Amount: \$100,000, Total Award Period Covered: 04/01/06-3/31/07.

PI: Prof. J. K. Furdyna; Co-PI: Prof. M. Dobrowolska and Prof. X. Liu. 
Title: Focused-Ion Beam (FIB) Nano-Fabrication and Characterization of Ferromagnetic Semiconductors (pending) Agency: University of Notre Dame

Total Award Amount: \$10,000, Total Award Period Covered: 1/01/2007-12/31/2008.

PI: Prof. X. Liu; Co-PI: Prof. M. Dobrowolska.

\section{SCHOLARSHIPS AND FELLOWSHIPS}

1992 Outstanding Student Scholarship, U. of Sci. \& Tech. of China

1993 Outstanding Student Scholarship, U. of Sci. \& Tech. of China

$1995 \quad$ Kwang-Hua Scholarship, Kwang-Hua Education Foundation

$1996 \quad$ Kwang-Hua Scholarship, Kwang-Hua Education Foundation

\section{RECENT AND CURRENT COLLABORATORS (48 MONTHS)}

Baxter, D. V. (Indiana University); Berciu, M. (University of British Columbia); Borchers, J. A. (National Institute of Standards and Technology); Chang, A. (Duke University); Chemla, D. S. (Lawrence Berkeley National Laboratory); Dubon, O. D. (UC-Berkeley); Gamelin, D. R. (University of Washington); Grimsditch, M. (Argonne National Laboratory); Hall, K. C. (Dalhousie University); Hyeon, T. (Seoul National University); Janko, B. (University of Notre Dame); Jaroszynski, J. (National High Magnet Field Lab); Kirby, B. J. (National Institute of Standards and Technology); Khodaparast, G. A. (Virginia Tech.); Lee, S. (Korea University); Lyanda-Geller, Y. B. (Purdue University); McNeil, L. (University of North Carolina); Merlin, R. (University of Michigan); Mihaly, G. (Budapest Technical University); Nadgorny, B. (Wayne State); Oh, E. (Chungnam University); Pascher, H. (Bayreuth University); Peiris, F. C. (Kenyon College); Rhyne, J. J. (Los Alamos National Laboratory); Rokhinson, L. P. (Purdue University); Storchak, V. G. (IV Kurchatov Atomic Energy Institute); Tolk, N. (Vanderbilt University); Twardowski, A. (Warsaw University); Walukiewicz, W. (Lawrence Berkeley National Laboratory); Wang, J. (Iowa State University); Wojtowicz, T. (Institute of Physics, Polish Academy of Sciences); Yee, K. J. (Seoul National University); Yu, K. M. (Lawrence Berkeley National Laboratory); Zhang, Y. H. (Arizona State University).

\section{OTHER EXPERIENCE}

- Participation as Grand Awards Judge for Physics in Intel International Science and Engineering Fair 2006

- Participation as Judge in the Northern Indiana Regional Science \& Engineering Fair 2006, 2008

- Elected president of Student Body of Modern Physics Department, U. of Sci. \& Tech. of China, 1992

\section{PROFESSIONAL SOCIETIES}

- American Physical Society

- The Minerals, Metals \& Materials Society (TMS)

- Sigma Xi, The Scientific Research Society

\section{PUBLICATIONS}

\section{Refereed Publications}

1. X. Liu, S. Shen, Z. Ge, W. L. Lim, M. Dobrowolska, J. K. Furdyna, and S. Lee, "Scaling relations between anomalous Hall and longitudinal transport coefficients in metallic (Ga,Mn)As films", Phys. Rev. B 83, 144421 (2011).

2. R. E. Pimpinella, A. M. Mintairov, X. Liu, T. H. Kosel, J. L. Merz, J. K. Furdyna, and M. Dobrowolska, "Optical measurements of single CdTe self-assembled quantum dots grown on $\mathrm{ZnTe} / \mathrm{GaSb}$ ", J. Vac. Sci. Technol. B 29, 03C119 (2011).

3. X. Liu, A. M. Mintairov, J. Herzog, F. Vietmeyer, R. E. Pimpinella, M. Kuno, J. L. Merz, T. H. Kosel, M. Dobrowolska, and J. K. Furdyna, "II-VI heterostructures obtained by encapsulation of colloidal CdSe nanowires by molecular beam epitaxy deposition of ZnSe", J. Vac. Sci. Technol. B 29, 03 C102 (2011).

4. Jinsik Shin, Shinhee Kim, Sangyeop Lee, Taehee Yoo, Hakjoon Lee, S. Khym, Sanghoon Lee, X. Liu, and J. K. Furdyna, "Asymmetry in the angular dependence of the switching field of GaMnAs film", J. Appl. Phys. 109, 07C308 (2011). 
5. K. Dziatkowski, X. Liu, J. K. Furdyna, and A. Twardowski, "Magnetic anisotropy in (Ga,Mn)As grown on vicinal GaAs: Effects of the orientation of microwave magnetic field", J. Appl. Phys. 109, 07C301 (2011).

6. J. Leiner, H. Lee, T. Yoo, Sanghoon Lee, B. J. Kirby, K. Tivakornsasithorn, X. Liu, J. K. Furdyna, and M. Dobrowolska, "Observation of antiferromagnetic interlayer exchange coupling in a

$\mathrm{Ga}_{1-\mathrm{x}} \mathrm{Mn}_{\mathrm{x}} \mathrm{As} / \mathrm{GaAs}: \mathrm{Be} / \mathrm{Ga}_{1-\mathrm{x}} \mathrm{Mn}_{\mathrm{x}} \mathrm{As}$ trilayer structure”, Phys. Rev. B 82, 195205 (2010).

7. Sangyeop Lee, Hakjoon Lee, Taehee Yoo, Sanghoon Lee, X. Liu, and J. K. Furdyna, "Influence of uniaxial anisotropy on the domain pinning fields of ferromagnetic $\mathrm{Ga}_{1-\mathrm{x}} \mathrm{Mn}_{\mathrm{x}} \mathrm{As}$ films", J. Appl. Phys. 108, 063910 (2010).

8. A. Winter, H. Pascher, H. Krenn, X. Liu, and J. K. Furdyna, "Interpretation of hysteresis loops of GaMnAs in the framework of the Stoner-Wohlfarth model", J. Appl. Phys. 108, 043921 (2010).

9. A. V. Scherbakov, A. S. Salasyuk, A. V. Akimov, X. Liu, M. Bombeck, C. Brüggemann, D. R. Yakovlev, V. F. Sapega, J. K. Furdyna, and M. Bayer, "Coherent Magnetization Precession in Ferromagnetic (Ga,Mn)As Induced by Picosecond Acoustic Pulses”, Phys. Rev. Lett. 105, 117204 (2010).

10. Sunjae Chung, Sanghoon Lee, J.-H. Chung, Taehee Yoo, Hakjoon Lee, B. Kirby, X. Liu, and J. K. Furdyna, "Giant magnetoresistance and long-range antiferromagnetic interlayer exchange coupling in (Ga,Mn)As/GaAs:Be multilayers", Phys. Rev. B 82, 054420 (2010).

11. Shinhee Kim, Hakjoon Lee, Taehee Yoo, Sangyeop Lee, Sanghoon Lee, X. Liu, and J. K. Furdyna, "Mapping of magnetic anisotropy in strained ferromagnetic semiconductor GaMnAs films", J. Appl. Phys. 107, 103911 (2010).

12. Hakjoon Lee, Sunjae Chung, Taehee Yoo, Sanghoon Lee, X. Liu, and J. K. Furdyna, "Reduction in the planar Hall resistance amplitude in the reversal process of Fe film with biaxial easy axes", J. Appl. Phys. 107, 09C508 (2010).

13. Taehee Yoo, S. Khym, Hakjoon Lee, Sunjae Chung, Sanghoon Lee, X. Liu, and J. K. Furdyna, "Asymmetry in the planar Hall resistance of Fe films grown on vicinal GaAs substrates", J. Appl. Phys. 107, 09C505 (2010).

14. Hyung-chan Kim, S. Khym, Sanghoon Lee, X. Liu, and J. K. Furdyna, "Magnetic anisotropy of $\mathrm{Ga}_{1-\mathrm{x}} \mathrm{Mn}_{\mathrm{x}} \mathrm{As}$ films with additional nonmagnetic donor doping", J. Appl. Phys. 107, 09C303 (2010).

15. Yungjun Kim, Sunjae Chung, Sanghoon Lee, X. Liu, and J. K. Furdyna, "Asymmetry in the reorientation process of magnetization for crossing the [1-10] and the [110] directions in $\mathrm{Ga}_{1-\mathrm{x}} \mathrm{Mn}_{\mathrm{x}} \mathrm{As}$ epilayers", J. Appl. Phys. 107, 09C304 (2010).

16. E. H. C. P. Sinnecker, G. M. Penello, T. G. Rappoport, M. M. Sant'Anna, D. E. R. Souza, M. P. Pires, J. K. Furdyna and X. Liu, "Ion-beam modification of the magnetic properties of $\mathrm{Ga}_{1-\mathrm{x}} \mathrm{Mn}_{\mathrm{x}} \mathrm{As}$ epilayers", Phys. Rev. B 81, 245203 (2010).

17. S. Chung, S. Lee, X. Liu, J. K. Furdyna, "Magnetization reorientation in $\mathrm{Ga}_{\mathrm{x}} \mathrm{Mn}_{1-\mathrm{x}} \mathrm{As}$ films: Planar Hall effect measurements", Phys. Rev. B 81, 155209 (2010).

18. R. E. Pimpinella, X. Liu, J. K. Furdyna, M. Dobrowolska, A. M. Mintairov, J. L. Merz, "Self-Assembled CdTe Quantum Dots Grown on ZnTe/GaSb", Journal of Electronic Materials 39, 992 (2010).

19. J. Qi, J. A. Yan, H. Park, A. Steigerwald, Y. Xu, S. N. Gilbert, X. Liu, J. K. Furdyna, S. T. Pantelides, and N. Tolk, "Mechanical and electronic properties of ferromagnetic $\mathrm{Ga}_{1-\mathrm{x}} \mathrm{Mn}_{\mathrm{x}}$ As using ultrafast coherent acoustic phonons", Phys. Rev. B 81, 115208 (2010).

20. H. Son, S. Chung, S. Yea, S. Kim, T. Yoo, S. Lee, X. Liu, and J. K. Furdyna, "Vertical gradient of magnetic anisotropy in the ferromagnetic semiconductor (Ga,Mn)As film", Appl. Phys. Lett. 96, 092105 (2010).

21. J. P. Zahn, A. Gamouras, S. March, X. Liu, J. K. Furdyna, and K. C. Hall, "Ultrafast studies of carrier and magnetization dynamics in GaMnAs", J. Appl. Phys. 107, 033908 (2010).

22. M. A. Mayer, P. R. Stone, N. Miller, H. M. Smith, III, O. D. Dubon, E. E. Haller, K. M. Yu, W. Walukiewicz, $\mathrm{X}$. Liu, and J. K. Furdyna, "Electronic structure of $\mathrm{Ga}_{1-\mathrm{x}} \mathrm{Mn}_{\mathrm{x}} \mathrm{As}$ analyzed according to hole-concentrationdependent measurements", Phys. Rev. B 81, 045205 (2010).

23. Y. Y. Zhou, X. Liu, J. K. Furdyna, M. A. Scarpulla, and O. D. Dubon, "Ferromagnetic resonance investigation of magnetic anisotropy in $\mathrm{Ga}_{1-\mathrm{x}} \mathrm{Mn}_{\mathrm{x}} \mathrm{As}$ synthesized by ion implantation and pulsed laser melting", Phys. Rev. B 80, 224403 (2009).

24. Y. Y. Zhou, X. Liu, J. K. Furdyna, M. A. Scarpulla, O. D. Dubon, "Ferromagnetic Resonance Study of $\mathrm{Ga}_{1-\mathrm{x}} \mathrm{Mn}_{\mathrm{x}} \mathrm{As}$ Fabricated on (311) GaAs Wafers by Mn Ion Implantation and Pulsed-Laser Melting", Journal of Superconductivity and Novel Magnetism 23, 87 (2010).

25. J. H. Yu, X. Liu, K. E. Kweon, J. Joo, J. Park, K. T. Ko, D. Lee, S. Shen, K. Tivakornsasithorn, J. S. Son, J.-H. Park, Y.-W. Kim, G. S. Hwang, M. Dobrowolska, J. K. Furdyna, T. Hyeon, "Giant Zeeman splitting in nucleation-controlled doped CdSe: $\mathrm{Mn}^{2+}$ quantum nanoribbons", Nature Materials 9, 47 (2010). 
26. J. Kim, S. Lee, S. Lee, X. Liu, J. K. Furdyna, "Investigation of domain pinning fields in ferromagnetic GaMnAs films using angular dependence of the planar Hall effect", Solid State Communications 150, 27 (2010).

27. T. Yoo, S. Khym, S. Yea, S. Chung, S. Lee, X. Liu, and J. K. Furdyna, "Four discrete Hall resistance states in single-layer Fe film for quaternary memory devices", Appl. Phys. Lett. 95, 202505 (2009).

28. C. Thurn, V. M. Axt, A. Winter, H. Pascher, H. Krenn, X. Liu, J. K. Furdyna, and T. Wojtowicz, "Origin of resonance structures in magneto-optical spectra of $\mathrm{InSb}$ and $\mathrm{In}_{1-\mathrm{x}} \mathrm{Mn}_{\mathrm{x}} \mathrm{Sb}$ ", Phys. Rev. B 80, 195210 (2009).

29. M. Frazier, J. G. Cates, J. A. Waugh, J. J. Heremans, M. B. Santos, X. Liu, and G. A. Khodaparast, "Photoinduced spin-polarized current in InSb-based structures", J. Appl. Phys. 106, 103513 (2009).

30. S. Chung, H. C. Kim, S. Lee, X. Liu, J. K. Furdyna, "The effect of carrier density on magnetic anisotropy of the ferromagnetic semiconductor (Ga, Mn)As", Solid State Communications 149, 1739 (2009).

31. A. Chernyshov, M. Overby, X. Liu, J. K. Furdyna, Y. Lyanda-Geller, and L. P. Rokhinson, "Evidence for reversible control of magnetization in a ferromagnetic material by means of spin-orbit magnetic field", Nature Physics 5, 656 (2009).

32. S. Shen, X. Liu, K. Tivakornsasithorn, Y. H. Cho, J. K. Furdyna, M. Dobrowolska, Y. H. Hwang, Y. H. Um, "Magneto-optical Studies of Spin Phenomena in CdMnTe Doped with Co and Cr", J. Elec. Mater. 38, 1554 (2009).

33. X. Zhang, S. Wang, D. Ding, X. Liu, J.-H. Tan, J. K. Furdyna, Y.-H. Zhang, D. J. Smith, "Structural Characterization of Integrated II-VI and III-V Heterostructures for Solar Cell Applications", J. Elec. Mater. 38, 1558 (2009).

34. H. Lee, S. Chung, S. Lee, X. Liu, J. K. Furdyna, "Temperature dependence of magnetization in GaMnAs film with critical strain", Solid State Communications 149, 1300 (2009).

35. H. Kim, H. Lee, S. J. Chung, S. Lee, Y. J. Cho, X. Liu, J. K. Furdyna, "Magneto-transport Properties of GaMnAs:Si Ferromagnetic Semiconductors", J. of the Korean Physical Society 55, 304 (2009).

36. D. M. Wang, Y. H. Ren, P. W. Jacobs, S. Fahy, X. Liu, J. K. Furdyna, V. F. Sapega, R. Merlin, "Observation of Insulating Nanoislands in Ferromagnetic GaMnAs", Phys. Rev. Lett. 102, 256401 (2009).

37. M. Berciu, R. Chakarvorty, Y. Y. Zhou, M. T. Alam, K. Traudt, R. Jakiela, A. Barcz, T. Wojtowicz, X. Liu, J. K. Furdyna, M. Dobrowolska, "Origin of Magnetic Circular Dichroism in GaMnAs: Giant Zeeman Splitting versus Spin Dependent Density of States", Phys. Rev. Lett. 102, 247202 (2009).

38. A. Winter, H. Pascher, M. Hofmayer, H. Krenn, T. Wojtowicz, X. Liu, J. K. Furdyna, "Kerr Rotation and Magnetic Circular Dichroism Spectra of Ferromagnetic InMnSb and InMnAs", Reviews on Advanced Materials Science 20, 92 (2009).

39. S. Wang, D. Ding, X. Liu, X.-B. Zhang, D. J. Smith, J. K. Furdyna, Y.-H. Zhang, "MBE growth of II-VI materials on GaSb substrates for photovoltaic applications", J. Crystal Growth 311, 2116 (2009).

40. D. G. Eshchenko, V. G. Storchak, E. Morenzoni, T. Prokscha, A. Suter, X. Liu, J. K. Furdyna, "Low energy mu SR studies of semiconductor interfaces", Physica B-Condensed Matter 404, 873 (2009).

41. Q. Song, K. H. Chow, R. I. Miller, I. Fan, M. D. Hossain, R. F. Kiefl, S. R. Kreitzman, C. D. P. Levy, T. J. Parolin, M. R. Pearson, Z. Salman, H. Saadaoui, M. Smadella, D. Wang, K. M. Yu, X. Liu, J. K. Furdyna, W. A. MacFarlane, "Beta-detected NMR study of the local magnetic field in epitaxial GaAs:Mn", Physica BCondensed Matter 404, 892 (2009).

42. S. Lee, J.-H. Chung, X. Liu, J. K. Furdyna, B. J. Kirby, "Ferromagnetic semiconductor GaMnAs", Materials Today 12, 14 (2009).

43. S. Shen, X. Liu, Y. J. Cho, J. K. Furdyna, M. Dobrowolska, Y. H. Hwang, Y. H. Um, "Ferromagnetic behavior of CdMnCrTe quaternary system”, Appl. Phys. Lett. 94, 142507 (2009).

44. T. Yoo, D. Shin, J. Kim, H. Kim, S. Lee, X. Liu, J. K. Furdyna, "Step feature observed in the angular dependence of magnetization switching fields in GaMnAs micro-device", Current Applied Physics 9, 773 (2009).

45. D. Y. Shin, S. Lee, X. Liu, J. K. Furdyna, "Monitoring of magnetization processes in GaMnAs ferromagnetic film by electrical transport measurement", J. Crystal Growth 311, 925 (2009).

46. S. Shen, X. Liu, J. K. Furdyna, M. Dobrowolska, Y. H. Hwang, and Y. H. Um, "Magneto-optical studies of $\mathrm{Cd}_{1-\mathrm{x}-\mathrm{y}} \mathrm{Mn}_{\mathrm{x}} \mathrm{Co}_{\mathrm{y}} \mathrm{Te}$ ", J. Appl. Phys. 105, 07A931 (2009).

47. H. Lee, S. Chung, S. Lee, X. Liu, and J. K. Furdyna, "Magnetotransport properties of GaMnAs based trilayer structures with different thicknesses of InGaAs spacer layer", J. Appl. Phys. 105, 07C505 (2009).

48. J. Kim, T. Yoo, S. Chung, S. Lee, X. Liu, and J. K. Furdyna, "Quantitative analysis of the angle dependence of planar Hall effect observed in ferromagnetic GaMnAs film”, J. Appl. Phys. 105, 07C501 (2009). 
49. A. Steigerwald, Y. Xu, J. Qi, J. Gregory, X. Liu, J. K. Furdyna, K. Varga, A. B. Hmelo, G. Lüpke, L. C. Feldman, and N. Tolk, "Semiconductor point defect concentration profiles measured using coherent acoustic phonon waves", Appl. Phys. Lett. 94, 111910 (2009).

50. J. Qi, Y. Xu, A. Steigerwald, X. Liu, J. K. Furdyna, I. E. Perakis, N. H. Tolk, "Ultrafast laser-induced coherent spin dynamics in ferromagnetic $\mathrm{Ga}_{1-\mathrm{x}} \mathrm{Mn}_{\mathrm{x}} \mathrm{As} / \mathrm{GaAs}$ structures", Phys. Rev. B 79, 085304 (2009).

51. J. K. Furdyna, X. Liu, Y. Y. Zhou, "Magnetic excitations in ferromagnetic semiconductors", Journal of Magnetism and Magnetic Materials, 321, 695 (2009).

52. J. Wang, I. Cotoros, D. S. Chemla, X. Liu, J. K. Furdyna, J. Chovan, and I. E. Perakis, "Memory effects in photoinduced femtosecond magnetization rotation in ferromagnetic GaMnAs", Appl. Phys. Lett. 94, 021101 (2009).

53. Y. J. Cho, K. M. Yu, X. Liu, W. Walukiewicz, and J. K. Furdyna, "Effects of donor doping on $\mathrm{Ga}_{1-\mathrm{x}} \mathrm{Mn}_{\mathrm{x}} \mathrm{As}$ ", Appl. Phys. Lett. 93, 262505 (2009).

54. Ingrid Cotoros, Jigang Wang, Xinyu Liu, Jacek K. Furdyna and Daniel S. Chemla, "Ultrafast Photoinduced Ferromagnetic Order in a Magnetic Semiconductor Heterostructure", in Ultrafast Phenomena XVI, Springer Series in Chemical Physics 92, P. Corkum, S. De Silvestri, K. A. Nelson, E. Riedle, and R. W. Schoenlein, eds. (Springer-Verlag, Berlin Heidelberg 2009), 197-199.

55. Ingrid Cotoros, Jigang Wang, Xinyu Liu, Jacek K. Furdyna, Jaroslav Chovan, Ilias E. Perakis and Daniel S. Chemla, "Memory Effects in Photo-induced Femtosecond Magnetization Rotation in a Ferromagnetic Semiconductor", in Ultrafast Phenomena XVI, Springer Series in Chemical Physics 92, P. Corkum, S. De Silvestri, K. A. Nelson, E. Riedle, and R. W. Schoenlein, eds. (Springer-Verlag, Berlin Heidelberg 2009), 212214.

56. J.-H. Chung, S. J. Chung, Sanghoon Lee, B. J. Kirby, J. A. Borchers, Y. J. Cho, X. Liu, and J. K. Furdyna, "Carrier-Mediated Antiferromagnetic Interlayer Exchange Coupling in Diluted Magnetic Semiconductor Multilayers $\mathrm{Ga}_{1-\mathrm{x}} \mathrm{Mn}_{\mathrm{x}} \mathrm{As} / \mathrm{GaAs}: B e ”$, Phys. Rev. Lett. 101, 237202 (2008).

57. Y. J. Cho, X. Liu, J. K. Furdyna, "Collapse of ferromagnetism in (Ga, Mn) As at high hole concentrations", Semicond. Sci. Technol. 23, 125010 (2008).

58. K. Alberi, K. M. Yu, P. R. Stone, O. D. Dubon, W. Walukiewicz, T. Wojtowicz, X. Liu, J. K. Furdyna, "Formation of Mn-derived impurity band in III-Mn-V alloys by valence band anticrossing", Phys. Rev. B 78, 075201 (2008).

59. J. Kim, D. Y. Shin, S. Lee, X. Liu, J. K. Furdyna, "Distribution of magnetic domain pinning fields in $\mathrm{Ga}_{1-}$ ${ }_{x} \mathrm{Mn}_{\mathrm{x}}$ As ferromagnetic films", Phys. Rev. B 78, 075309 (2008).

60. Y. J. Cho, M. A. Scarpulla, Y. Y. Zhou, Z. Ge, X. Liu, M. Dobrowolska, K. M. Yu, O. D. Dubon, J. K. Furdyna, "Magnetic anisotropy of ferromagnetic $\mathrm{Ga}_{1-\mathrm{x}} \mathrm{Mn}_{\mathrm{x}} \mathrm{As}$ formed by $\mathrm{Mn}$ ion implantation and pulsed-laser melting", J. Appl. Phys. 104, 043902 (2008).

61. S.-Y. Yea, S. J. Chung, H. Son, D. Y. Shin, S. Lee, X. Liu, J. K. Furdyna, "Effect of chemical etching on magnetic anisotropy of ferromagnetic GaMnAs films studied by planar Hall effect", Solid State Communications 147, 309 (2008).

62. B. Brodowska, I. Kuryliszyn-Kudelska, T. Wojtowicz, M. Arciszewska, W. Dobrowolski, E. I. Slynko, V. E. Slynko, X. Liu, and J. K. Furdyna, "Magnetoresistance near the ferromagnetic-paramagnetic phase transition in magnetic semiconductors", Appl. Phys. Lett. 93, 042113 (2008).

63. K. C. Hall, J. P. Zahn, A. Gamouras, S. March, J. L. Robb, X. Liu, and J. K. Furdyna, "Ultrafast optical control of coercivity in GaMnAs", Appl. Phys. Lett. 93, 032504 (2008).

64. V. G. Storchak, D. G. Eshchenko, E. Morenzoni, T. Prokscha, A. Suter, X. Liu, and J. K. Furdyna, "Spatially Resolved Inhomogeneous Ferromagnetism in (Ga,Mn)As Diluted Magnetic Semiconductors: A Microscopic Study by Muon Spin Relaxation", Phys. Rev. Lett. 101, 027202 (2008).

65. A. Geresdi, A. Halbritter, M. Csontos, S. Csonka, G. Mihaly, T. Wojtowicz, X. Liu, B. Janko, J. K. Furdyna, "Nanoscale spin polarization in the dilute magnetic semiconductor (In,Mn)Sb", Phys. Rev. B 77, 233304 (2008).

66. M. Overby, A. Chernyshov, L. P. Rokhinson, X. Liu, and J. K. Furdyna, "GaMnAs-based hybrid multiferroic memory device", Appl. Phys. Lett. 92, 192501 (2008).

67. C. Gould, S. Mark, K. Pappert, R. G. Dengel, J. Wenisch, R. P. Campion, A. W. Rushforth, D. Chiba, Z. Li, X. Liu, W. Van Roy, H. Ohno, J. K. Furdyna, B. Gallagher, K. Brunner, G. Schmidt, L. W. Molenkamp, “An extensive comparison of anisotropies in MBE grown (Ga, Mn)As material", New Journal of Physics 10, 055007 (2008).

68. S. Shen, X. Liu, Z. Ge, J. K. Furdyna, M. Dobrowolska, and J. Jaroszynski, "Scaling of the anomalous Hall effect in low Mn concentration (Ga,Mn)As", J. Appl. Phys. 103, 07D134 (2008). 
69. Y. J. Cho, X. Liu, and J. K. Furdyna, "Vanishing of ferromagnetic order in (Ga,Mn)As films at high hole concentrations: beyond the mean field Zener model", J. Appl. Phys. 103, 07D132 (2008).

70. Taehee Yoo, Dongyun Shin, Jungtaek Kim, Hyungchan Kim, Sanghoon Lee, X. Liu, and J. K. Furdyna, "Effect of shape anisotropy on the magnetization reversal process of (Ga,Mn)As ferromagnetic semiconductors", J. Appl. Phys. 103, 07D119 (2008).

71. Sun-Young Yea, Sun-Jae Chung, Hyunji Son, Sanghoon Lee, X. Liu, and J. K. Furdyna, "Thickness dependence of magnetic domain pinning energy in GaMnAs ferromagnetic semiconductor films", J. Appl. Phys. 103, 07D118 (2008).

72. Hyunji Son, Sun-jae Chung, Sun-young Yea, Sanghoon Lee, X. Liu, and J. K. Furdyna, "Quantitative investigation of the magnetic anisotropy in GaMnAs film by using Hall measurement", J. Appl. Phys. 103, 07F313 (2008).

73. B. J. Kirby, J. A. Borchers, X. Liu, Z. Ge, Y. J. Cho, M. Dobrowolska, and J. K. Furdyna, "Magnetization reversal of $\mathrm{Ga}_{1-x} \mathrm{Mn}_{x}$ As layers separated by a nonmagnetic spacer", J. Appl. Phys. 103, 07D116 (2008).

74. Jungtaek Kim, D. Y. Shin, Taehee Yoo, Hyungchan Kim, Sanghoon Lee, X. Liu, and J. K. Furdyna, "Single and multidomain characteristics of GaMnAs investigated by magnetotransport measurements", J. Appl. Phys. 103, 07D101 (2008).

75. Y. D. Glinka, N. H. Tolk, X. Liu, Y. Sasaki, and J. K. Furdyna, "Hot-phonon-assisted absorption at semiconductor heterointerfaces monitored by pump-probe second-harmonic generation", Phys. Rev. B 77, 113310 (2008).

76. G. Mihály, M. Csontos, S. Bordács, I. Kézsmárki, T. Wojtowicz, X. Liu, B. Jankó, and J. K. Furdyna "Anomalous Hall Effect in the (In,Mn)Sb Dilute Magnetic Semiconductor", Phys. Rev. Lett. 100, 107201 (2008).

77. Y. D. Glinka, N. H. Tolk, X. Liu, Y. Sasaki, and J. K. Furdyna, "Electro-optic nature of ultrafast pump-probe reflectivity response from multilayer semiconductor heterostructures", J. Appl. Phys. 103, 043708 (2008).

78. M. Frazier, R. N. Kini, K. Nontapot, G. A. Khodaparast, T. Wojtowicz, X. Liu, and J. K. Furdyna, "Time resolved magneto-optical studies of ferromagnetic InMnSb films", Appl. Phys. Lett. 92, 061911 (2008).

79. R. Beaulac, P. I. Archer, X. Liu, S. Lee, G. M. Salley, M. Dobrowolska, J. K. Furdyna, D. R. Gamelin, "Spinpolarizable excitonic luminescence in colloidal $\mathrm{Mn}^{2+}$-doped CdSe quantum dots", Nano Letters 8, 1197 (2008).

80. S. J. Chung, D. Y. Shin, H. Kim, S. Lee, X. Liu, and J. K. Furdyna, "Magneto-transport properties of a GaMnAs-based ferromagnetic semiconductor trilayer structure grown on a ZnMnSe buffer", J. Elec. Mater. 37, $912(2008)$.

81. S. J. Chung, D. Y. Shin, H. Son, S. Lee, X. Liu, and J. K. Furdyna, "Time stability of multi-domain states formed in the magnetization reversal process of GaMnAs film", Solid State Comm. 143, 232 (2007).

82. Raghava P. Panguluri, B. Nadgorny, T. Wojtowicz, X. Liu, and J. K. Furdyna, "Inelastic scattering and spin polarization in dilute magnetic semiconductor (Ga,Mn)Sb", Appl. Phys. Lett. 91, 252502 (2007).

83. Y. D. Glinka, N. H. Tolk, X. Liu, Y. Sasaki, and J. K. Furdyna, "Spatially resolved pump-probe second harmonic generation study of multilayer semiconductor heterostructures", Appl. Phys. Lett. 91, 231104 (2007).

84. B. J. Kirby, J. A. Borchers, X. Liu, Z. Ge, Y. J. Cho, M. Dobrowolska, and J. K. Furdyna, "Definitive evidence of interlayer coupling between $\mathrm{Ga}_{1-x} \mathrm{Mn}_{x} \mathrm{As}$ layers separated by a nonmagnetic spacer", Phys. Rev. B 76, 205316 (2007).

85. R. Chakarvorty, S. Shen, K. J. Yee, T. Wojtowicz, R. Jakiela, A. Barcz, X. Liu, J. K. Furdyna, and M. Dobrowolska, "Common origin of ferromagnetism and band edge Zeeman splitting in GaMnAs at low Mn concentrations", Appl. Phys. Lett. 91, 171118 (2007).

86. L. P. Rokhinson, Y. Lyanda-Geller, Z. Ge, S. Shen, X. Liu, M. Dobrowolska, and J. K. Furdyna, "Weak localization in $\mathrm{Ga}_{1-x} \mathrm{Mn}_{x} \mathrm{As}$ : Evidence of impurity band transport", Phys. Rev. B 76, 161201(R) (2007).

87. Z. Ge, Y. Y. Zhou, Y.-J. Cho, X. Liu, J. K. Furdyna, and M. Dobrowolska, "Investigation of magnetic and electronic coupling between two $(\mathrm{Ga}, \mathrm{Mn}) \mathrm{As}$ layers in $(\mathrm{Ga}, \mathrm{Mn}) \mathrm{As} / \mathrm{GaAs} /(\mathrm{Ga}, \mathrm{Mn}) \mathrm{As}$ magnetic tunnel junctions", Appl. Phys. Lett. 91, 152109 (2007).

88. K. Dziatkowski, Z. Ge, X. Liu, J. K. Furdyna, B. Clerjaud, A. Twardowski, "Ferromagnetic resonance study of exchange coupled (Ga,Mn)As/GaAs/(Ga,Mn)As heterostructures", Acta Physica Polonica A, 112, 227-232 (2007).

89. J. Qi, Y. Xu, N. H. Tolk, X. Liu, J. K. Furdyna, and I. E. Perakis, "Coherent magnetization precession in GaMnAs induced by ultrafast optical excitation”, Appl. Phys. Lett. 91, 112506 (2007).

90. Z. Ge, W. L. Lim, R. Chakarvorty, S. Shen, X. Liu, J. K. Furdyna, and M. Dobrowolska, "Electroluminescence studies of (Ga,Mn)As-based p-i-n structures”, J. Appl. Phys. 102, 054507 (2007). 
91. D. Y. Shin, S. J. Chung, Sanghoon Lee, X. Liu, and J. K. Furdyna, "Temperature dependence of magnetic anisotropy in ferromagnetic (Ga,Mn)As films: Investigation by the planar Hall effect”, Phys. Rev. B 76, 035327 (2007).

92. Z. L. Fang, P. Wu, N. Kundtz, A. M. Chang, X. Y. Liu, and J. K. Furdyna, "Spin-dependent resonant tunneling through $6 \mu \mathrm{m}$ diameter double barrier resonant tunneling diode", Appl. Phys. Lett. 91, 022101 (2007).

93. D. M. Wang, Y. H. Ren, X. Liu, J. K. Furdyna, M. Grimsditch, and R. Merlin, "Light-induced magnetic precession in (Ga,Mn)As slabs: Hybrid standing-wave Damon-Eshbach modes" Phys. Rev. B 75, 233308 (2007).

94. J. Wang, I. Cotoros, K. M. Dani, X. Liu, J. K. Furdyna, and D. S. Chemla, "Ultrafast Enhancement of Ferromagnetism via Photoexcited Holes in GaMnAs", Phys. Rev. Lett. 98, 217401 (2007).

95. X. Liu, Y. Y. Zhou, and J. K. Furdyna, “Angular dependence of spin-wave resonances and surface spin pinning in ferromagnetic (Ga,Mn) As films”, Phys. Rev. B 75, 195220 (2007).

96. Sanghoon Lee, D. Y. Shin, S. J. Chung, X. Liu, and J. K. Furdyna, "Tunable quaternary states in ferromagnetic semiconductor GaMnAs single layer for memory devices”, Appl. Phys. Lett. 90, 152113 (2007).

97. K. Nontapot, R. N. Kini, A. Gifford, T. R. Merritt, G. A. Khodaparast, T. Wojtowicz, X. Liu, and J. K. Furdyna, "Relaxation of photoinduced spins and carriers in ferromagnetic InMnSb films", Appl. Phys. Lett. 90, 143109 (2007).

98. Kang-Jeon Han, Ji-Hee Kim, Ki-Ju Yee, X. Liu, J. K. Furdyna, and F. Hache, "Magnetization-induced optical nonlinearity in ferromagnetic GaMnAs", J. Appl. Phys. 101, 063519 (2007).

99. D. Y. Shin, S. J. Chung, Sanghoon Lee, X. Liu, and J. K. Furdyna, "Stable Multidomain Structures Formed in the Process of Magnetization Reversal in GaMnAs Ferromagnetic Semiconductor Thin Films", Phys. Rev. Lett. 98, 047201 (2007).

100. K. Alberi, J. Wu, W. Walukiewicz, K. M. Yu, O. D. Dubon, S. P. Watkins, C. X. Wang, X. Liu, Y.-J. Cho, and J. Furdyna, "Valence-band anticrossing in mismatched III-V semiconductor alloys", Phys. Rev. B 75, 045203 (2007).

101. Z. Ge, W. L. Lim, S. Shen, Y. Y. Zhou, X. Liu, J. K. Furdyna, and M. Dobrowolska, "Magnetization reversal in $(\mathrm{Ga}, \mathrm{Mn}) \mathrm{As} / \mathrm{MnO}$ exchange-biased structures: Investigation by planar Hall effect", Phys. Rev. B 75, 014407 (2007).

102. D. M. Wang, Y. H. Ren, X. Liu, J. K. Furdyna, M. Grimsditch, and R. Merlin, "Ultrafast optical study of magnons in the ferromagnetic semiconductor GaMnAs", Superlattices and Microstructures 41, 372 (2007).

103. Z. Ge, W.-L. Lim, Y. J. Cho, X. Liu, J. K. Furdyna, M. Dobrowolska, "Temperature dependence of exchange bias and coercivity in (Ga,Mn)As-MnO bilayer structures”, IEEE Trans. Magn. 43, 3013 (2007).

104. B. J. Kirby, M. R. Fitzsimmons, J. A. Borchers, Z. Ge, X. Liu, J. K. Furdyna, "Pinned spin depth profile of an oxidized-Mn/ $\mathrm{Ga}_{1-\mathrm{x}} \mathrm{Mn}_{\mathrm{x}} \mathrm{As}$ exchange bias bilayer - The effects of overannealing”, IEEE Trans. Magn. 43, 3016 (2007).

105. Y. Y. Zhou, Y. J. Cho, Z. Ge, X. Liu, M. Dobrowolska, and J. K. Furdyna, "Magnetic anisotropy, spin pinning, and exchange constants of (Ga,Mn)As films", IEEE Trans. Magn. 43, 3019 (2007).

106. D. Y. Shin, S. J. Chung, S. Lee, X. Liu, and J. K. Furdyna, "Precise investigation of domain pinning energy in GaMnAs using planar Hall effect and magnetoresistance measurements", IEEE Trans. Magn. 43, 3025 (2007).

107. R. Chakarvorty, Y. Y. Zhou, Y. J. Cho, X. Liu, R. Jakiela, A. Barcz, J. K. Furdyna, and M. Dobrowolska, "Determination of Mn acceptor compensation in MBE-grown GaMnAs via magnetic circular dichroism (MCD)", IEEE Trans. Magn. 43, 3031 (2007).

108. X. Liu, J. K. Furdyna, M. Dobrowolska, W. L. Lim, C. Xie, Y. J. Cho, "Unique properties of magnetotransport in GaMnAs films grown on vicinal and high-index planes”, J. Phys.: Condens. Matter 19, 165205 (2007).

109. K. J. Han, J. H. Kim, D. W. Jang, K. J. Yee, X. Liu, J. K. Furdyna, and Y. S. Lim, "Control of coherent phonon decay in GaAs by using a secondary pump pulse", Journal of the Korean Physical Society 50, 781 (2007).

110. J. H. Kim, K. J. Han, D. W. Jang, K. J. Yee, X. Liu, J. K. Furdyna, "Spin-polarized photoreflectance in ferromagnetic GaMnAs", Journal of the Korean Physical Society 50, 819 (2007).

111. T. Kim, S. J. Chung, D. Y. Shin, I. S. Choi, S. Lee, X. Liu, and J. K. Furdyna, "Strain-engineered magnetic anisotropy of GaMnAs ferromagnetic semiconductors", Journal of the Korean Physical Society 50, 829 (2007).

112. B. J. Kirby, J. A. Borchers, J. J. Rhyne, K. V. O'Donovan, S. G. E. te Velthuis, S. Roy, Cecilia Sanchez-Hanke, T. Wojtowicz, X. Liu, W. L. Lim, M. Dobrowolska, and J. K. Furdyna, "Magnetic and chemical nonuniformity in $\mathrm{Ga}_{1-x} \mathrm{Mn}_{x} \mathrm{As}$ films as probed by polarized neutron and X-ray reflectometry", Phys. Rev. B 74, 245304 (2006).

113. J. K. Miller, J. Qi, Y. Xu, Y. J. Cho, X. Liu, J. K. Furdyna, I. Perakis, T. V. Shahbazyan, N. Tolk, "Nearbandgap wavelength dependence of long-lived traveling coherent longitudinal acoustic phonons in GaSb-GaAs heterostructures", Phys. Rev. B 74, 113313 (2006). 
114. K. Dziatkowski, Z. Ge, X. Liu, J. K. Furdyna, A. Twardowski, “Asymmetric magnetization reversal in the exchange-biased $\mathrm{MnO} /(\mathrm{Ga}, \mathrm{Mn})$ As heterostructure studied by ferromagnetic resonance", Acta Physica Polonica A, 110, 319-324 (2006).

115. X. Liu, J. K. Furdyna, "Ferromagnetic resonance of $\mathrm{Ga}_{1-x} \mathrm{Mn}_{x} \mathrm{As}$ dilute magnetic semiconductors", J. Phys.: Condens. Matter 18, R245-279 (2006).

116. W. L. Lim, X. Liu, K. Dziatkowski, Z. Ge, S. Shen, J. K. Furdyna, M. Dobrowolska, "Effect of magnetic anisotropy on the transverse planar Hall resistance of $\mathrm{Ga}_{1-x} \mathrm{Mn}_{x} \mathrm{As}$ films grown on vicinal GaAs substrates", Phys. Rev. B 74, 045303 (2006).

117. S. Balascuta, X. Liu, D. V. Baxter, J. Carini, T. Wojtowicz, Y. Sasaki, J. K. Furdyna, M. Chipara, "Ferromagnetic resonance investigations on $\mathrm{Ga}_{0.965} \mathrm{Mn}_{0.035} \mathrm{As}$ film" J. Appl. Phys. 99, 113908 (2006).

118. X. Liu, M. Dobrowolska, J. K. Furdyna, S. Lee, "Temperature-dependent photoluminescence of vertically stacked self-assembled CdSe quantum dots in ZnSe", Physica E 32, 65-68 (2006).

119. W. L. Lim, X. Liu, K. Dziatkowski, Z. Ge, S. Shen, J. K. Furdyna, M. Dobrowolska, "Investigation of magnetocrystalline anisotropy by planar Hall effect in GaMnAs epilayers grown on vicinal GaAs substrates", J. Appl. Phys. 99, 08D505 (2006).

120. I. S. Choi, S. Y. An, S. J. Chung, S. Lee, X. Liu, J. K. Furdyna, "Transport properties of ferromagnetic GaMnAs interfaced with paramagnetic ZnMnSe in the form of bilayer structures" J. Appl. Phys. 99, 08 D512 (2006).

121. K. Dziatkowski, Z. Ge, X. Liu, and J. K. Furdyna, "Identification of unidirectional anisotropy in exchangebiased MnO/GaMnAs bilayers using ferromagnetic resonance”, Appl. Phys. Lett. 88, 142513 (2006).

122. K. J. Yee, D. Lee, X. Liu, M. Dobrowolska, J. K. Furdyna, K. G. Lee, D. S. Kim, and Y. S. Lim, "Dynamic longitudinal-optical phonon decay via transient electron-phonon interactions in low-temperature-grown GaAs", Appl. Phys. Lett. 88, 121904 (2006).

123. R. Bacewicz, A. Twaróg, A. Malinowska, T. Wojtowicz, X. Liu, J.K. Furdyna, "Local structure of Mn in (Ga,Mn)As probedby X-ray absorption spectroscopy”, J. Phys. Chem. Solids 66, 2004 (2005).

124. K. J. Yee, D. Lee, X. Liu, W. L. Lim, M. Dobrowolska, J. K. Furdyna, Y. S. Lim, K. G. Lee, Y. H. Ahn, and D. S. Kim, "Optical studies of carrier and phonon dynamics in $\mathrm{Ga}_{1-x} \mathrm{Mn}_{x} \mathrm{As}$ ", J. Appl. Phys. 98, 113509 (2005).

125. M. Csontos, T. Wojtowicz, X. Liu, M. Dobrowolska, B. Jankó, G. Mihály, J. K. Furdyna, "Magnetic scattering of spin polarized carriers in (In,Mn)Sb dilute magnetic semiconductor", Phys. Rev. Lett. 95, 227203 (2005).

126. L. V. Titova, M. Kutrowski, X. Liu, R. Chakarvorty, W. L. Lim, T. Wojtowicz, J. K. Furdyna, and M. Dobrowolska, "Competition between cubic and uniaxial anisotropy in $\mathrm{Ga}_{1-x} \mathrm{Mn}_{x} \mathrm{As}$ in the low-Mnconcentration limit", Phys. Rev. B 72, 165205 (2005).

127. X. Liu, W. L. Lim, L. V. Titova, M. Dobrowolska, J. K. Furdyna, M. Kutrowski, and T. Wojtowicz, "Perpendicular magnetization reversal, magnetic anisotropy, multistep spin switching, and domain nucleation and expansion in $\mathrm{Ga}_{1-x} \mathrm{Mn}_{x}$ As films", J. Appl. Phys. 98, 063904 (2005).

128. S. Lee, S. J. Chung, X. Liu, J. K. Furdyna, "Doping effect on the properties of III-V ferromagnetic semiconductor GaMnAs epilayers and their superlattices", J. Korean Physical Society 47, 444 (2005).

129. R. P. Panguluri, K. C. Ku, T. Wojtowicz, X. Liu, J. K. Furdyna, Y. B. Lyanda-Geller, N. Samarth, and B. Nadgorny, "Andreev reflection and pair-breaking effects at the superconductor/magnetic semiconductor interface”, Phys. Rev. B 72, 054510 (2005).

130. R. Lang, A. Winter, H. Pascher, H. Krenn, X. Liu, and J. K. Furdyna, "Polar Kerr effect studies of Ga $\mathrm{G}_{1-x} \mathrm{Mn}_{x} \mathrm{As}$ epitaxial films", Phys. Rev. B 72, 024430 (2005).

131. Z. J. Weber, F. C. Peiris, X. Liu, and J. K. Furdyna, "Dielectric functions of molecular-beam-epitaxy-grown $\mathrm{Ga}_{1-x} \mathrm{Mn}_{x}$ As thin films”, J. Vac. Sci. Technol. B 23, 1313 (2005).

132. M. Csontos, G. Mihály, B. Jankó, T. Wojtowicz, X. Liu, J. K. Furdyna, "Pressure-induced ferromagnetism in (In,Mn)Sb dilute magnetic semiconductor", Nature Materials 4, 447 (2005).

133. S. J. Chung, S. Lee, I. W. Park, X. Liu, and J. K. Furdyna, "Effect of low temperature annealing on the magnetic properties of $\mathrm{Ga}_{1-x} \mathrm{Mn}_{x} \mathrm{As} / \mathrm{GaAs}$ superlattices", Journal of Superconductivity 18, 93 (2005).

134. K. J. Yee, Y. S. Lim, X. Liu, W. L. Lim, D. S. Kim, M. Dobrowolska, and J. K. Furdyna, "Coherent optical phonon oscillations in GaMnAs", Journal of Superconductivity 18, 115 (2005).

135. K. J. Yee, R. Chakarvorty, W. L. Lim, X. Liu, M. Kutrowski, L. V. Titova, T. Wojtowicz, J. K. Furdyna and M. Dobrowolska, "Observation of combined ferromagnetic/paramagnetic phase in $\mathrm{Ga}_{1-x} \mathrm{Mn}_{x} \mathrm{As}$ by magnetic circular dichroism”, Journal of Superconductivity 18, 131 (2005).

136. X. Liu, W. L. Lim, Z. Ge, S. Shen, M. Dobrowolska, J. K. Furdyna, T. Wojtowicz, K. M. Yu, and W. Walukiewicz, "Strain-engineered ferromagnetic $\mathrm{In}_{1-x} \mathrm{Mn}_{x} \mathrm{As}$ films with in-plane easy axis", Appl. Phys. Lett. 86, $112512(2005)$. 
137. G. B. Kim, M. Na, G. Acbas, B. D. Mccombe, S. Wang, M. Cheon, H. Luo, X. Liu, Y. Sasaki, and J. K. Furdyna, "High-field magnetotransport studies of ferromagnetic GaAs/Mn digital alloys", International Journal of Modern Physics B 18, 3735 (2004).

138. B. J. Kirby, J. A. Borchers, J. J. Rhyne, K. V. O'Donovan, T. Wojtowicz, X. Liu, Z. Ge, S. Shen, and J. K. Furdyna, "Effects of capping on the $\mathrm{Ga}_{1-x} \mathrm{Mn}_{x}$ As magnetic depth profile", Appl. Phys. Lett. 86, 072506 (2005).

139. K. M. Yu, W. Walukiewicz, T. Wojtowicz, J. Denlinger, M. A. Scarpulla, X. Liu, and J. K. Furdyna, “Effect of film thickness on the incorporation of Mn interstitials in $\mathrm{Ga}_{1-x} \mathrm{Mn}_{x} \mathrm{As}$ ", Appl. Phys. Lett. 86, 042102 (2005).

140. X. Liu, W. L. Lim, M. Dobrowolska, J. K. Furdyna, and T. Wojtowicz, "Ferromagnetic resonance study of the free-hole contribution to magnetization and magnetic anisotropy in modulation-doped $\mathrm{Ga}_{1-x} \mathrm{Mn}_{x} \mathrm{As} / \mathrm{Ga}_{1-}$ ${ }_{y} \mathrm{Al}_{y} \mathrm{As}:$ Be", Phys. Rev. B 71, 035307 (2005).

141. M. Kutrowski, T. Wojtowicz, G. Cywinski, L. V. Titova, E. Martin, X. Liu, J. K. Furdyna, and M. Dobrowolska, "Observation of photoluminescence related to Lomer-Cottrell-like dislocations in ZnSe epilayers grown on in situ cleaved (110)GaAs surfaces”, J. Appl. Phys. 97, 013519 (2005).

142. I. S. Choi, S. H. Nam, H. J. Lee, S. Lee, S. Y. An, W. L. Lim, X. Liu, and J. K. Furdyna, "Magnetotransport properties of GaMnAs/ZnMnSe double-layer systems”, J. Korean Physical Society 45, S554 (2004).

143. S. U. Yuldashev, H. Im, T. W. Kang, S. H. Lee, Y. Sasaki, X. Liu, J. K. Furdyna, and Y. D. Woo, “Origin of resistivity peak near the Curie temperature and magnetoresistance in $\mathrm{Ga}_{1-x} \mathrm{Mn}_{x} \mathrm{As}$ epitaxial layers", J. Korean Physical Society 45, S572 (2004).

144. Y. J. Yoon, S. J. Chung, H. J. Lee, S. Lee, S. Y. An, X. Liu, and J. K. Furdyna, "Effect of $p$-type buffer layer on the properties of GaMnAs ferromagnetic semiconductors", J. Korean Physical Society 45, S720 (2004).

145. J. K. Furdyna, T. Wojtowicz, X. Liu, K. M. Yu, W. Walukiewicz, I. Vurgaftman, and J. R. Meyer, "Fermi level effects on $\mathrm{Mn}$ incorporation in modulation-doped ferromagnetic $\mathrm{III}_{1-x} \mathrm{Mn}_{x} \mathrm{~V}$ heterostructures", J. Phys.-Condens. Matter 16, S5499 (2004).

146. T. Wojtowicz, J. K. Furdyna, X. Liu, K. M. Yu, and W. Walukiewicz, "Electronic effects determining the formation of ferromagnetic $\mathrm{III}_{1-\mathrm{x}} \mathrm{Mn}_{\mathrm{x}} \mathrm{V}$ alloys during epitaxial growth", Physica E, 25, 171 (2004).

147. U. Welp, V. K. Vlasko-Vlasov, A. Menzel, H. D. You, X. Liu, J. K. Furdyna, and T. Wojtowicz, "Uniaxial inplane magnetic anisotropy of $\mathrm{Ga}_{1-x} \mathrm{Mn}_{x} \mathrm{As}$ ”, Appl. Phys. Lett. 85, 260 (2004).

148. A. Wolos, M. Kaminska, M. Palczewska, A. Twardowski, X. Liu, T. Wojtowicz, and J. K. Furdyna, "Properties of arsenic antisite defects in $\mathrm{Ga}_{1-x} \mathrm{Mn}_{x} \mathrm{As}$ ", J. Appl. Phys. 96, 530 (2004)

149. K. M. Yu, W. Walukiewicz, T. Wojtowicz, W. L. Lim, X. Liu, M. Dobrowolska, J. K. Furdyna, "Lattice location of $\mathrm{Mn}$ and fundamental Curie temperature limit in ferromagnetic $\mathrm{Ga}_{1-\mathrm{x}} \mathrm{Mn}_{\mathrm{x}} \mathrm{As}$ ", Nuclear Instruments \& Methods in Physics Research, Section B - Beam Interactions with Materials and Atoms 219-20, 636-641 (2004).

150. X. Liu and J. K. Furdyna, “Optical dispersion of ternary II-VI semiconductor alloys”, J. Appl. Phys. 95, 7754 (2004).

151. R. P. Panguluri, B. Nadgorny, T. Wojtowicz, W. L. Lim, X. Liu, and J. K. Furdyna, "Measurement of spin polarization by Andreev reflection in ferromagnetic $\mathrm{In}_{1-x} \mathrm{Mn}_{x} \mathrm{Sb}$ epilayers", Appl. Phys. Lett. 84, 4947 (2004).

152. S. J. Chung, S. Lee, I. W. Park, X. Liu, and J. K. Furdyna, "Possible indication of interlayer exchange coupling in GaMnAs/GaAs ferromagnetic semiconductor superlattices”, J. Appl. Phys. 95, 7402 (2004).

153. S. U. Yuldashev, Y. Kim, N. Kim, H. Im, T. W. Kang, S. Lee, Y. Sasaki, X. Liu, and J . K. Furdyna, "Effect of interlayer exchange coupling on the Curie temperature in $\mathrm{Ga}_{1-x} \mathrm{Mn}_{x}$ As trilayer structures", Japanese J. APPL. Phys. 1 43, 2093 (2004).

154. D. Ruzmetov, J. Scherschligt, D. V. Baxter, T. Wojtowicz, X. Liu, Y. Sasaki, J. K. Furdyna, K. M. Yu, and W. Walukiewicz, "High-temperature Hall effect in $\mathrm{Ga}_{1-x} \mathrm{Mn}_{x} \mathrm{As}$ ", Phys. Rev. B 69, 155207 (2004).

155. K. M. Yu, W. Walukiewicz, T. Wojtowicz, W. L. Lim, X. Liu, M. Dobrowolska, and J. K. Furdyna, "Direct evidence of the Fermi-energy-dependent formation of $\mathrm{Mn}$ interstitials in modulation-doped $\mathrm{Ga}_{1-y} \mathrm{Al}_{y} \mathrm{As}_{\mathrm{S}} / \mathrm{Ga}_{1-}$ ${ }_{x} \mathrm{Mn}_{x} \mathrm{As} / \mathrm{Ga}_{1-y} \mathrm{Al}_{y} \mathrm{As}$ heterostructures", Appl. Phys. Lett. 84, 4325 (2004).

156. T. G. Rappoport, P. Redliñski, X. Liu, G. Zaránd, J. K. Furdyna, and B. Jankó, “Anomalous behavior of spinwave resonances in $\mathrm{Ga}_{1-x} \mathrm{Mn}_{x}$ As thin films", Phys. Rev. B 69, 125213 (2004).

157. B. J. Kirby, J. A. Borchers, J. J. Rhyne, S. G. E. te Velthuis, A. Hoffmann, K. V. O'Donovan, T. Wojtowicz, X. Liu, W. L. Lim, and J. K. Furdyna, "Annealing-dependent magnetic depth profile in $\mathrm{Ga}_{1-x} \mathrm{Mn}_{x} \mathrm{As}$ ”, Phys. Rev. B 69, 081307 (2004).

158. J. Sinova, T. Jungwirth, X. Liu, Y. Sasaki, J. K. Furdyna, W. A. Atkinson, and A. H. MacDonald, "Magnetization relaxation in (Ga,Mn)As ferromagnetic semiconductors", Phys. Rev. B 69, 085209 (2004).

159. J. Franz, F. C. Peiris, X. Liu, U. Bindley and J. K. Furdyna, "Determination of the dielectric functions of MBEgrown $\mathrm{Zn}_{1-x} \mathrm{Mg}_{x} \mathrm{Te}$ II-VI semiconductor alloys”, Phys. Status Solidi B 241, 507 (2004). 
160. L. V. Titova, G. Cywinski, M. Kutrowski, T. Wojtowicz, X. Liu, J. K. Furdyna and M. Dobrowolska, “ZnCdSe quantum structures by (110)-cleaved-edge overgrowth: MBE growth and $\mu$-PL characterization", Phys. Status Solidi B 241, 519 (2004).

161. T. Wojtowicz, W.L. Lim, X. Liu, G. Cywinski, M. Kutrowski, L.V. Titova, K. Yee, M. Dobrowolska, J.K. Furdyna, K.M. Yu, W. Walukiewicz, G.B. Kim, M. Cheon, X. Chen, S.M. Wang, H. Luo, I. Vurgaftman, and J.R. Meyer, "Growth and properties of ferromagnetic $\mathrm{In}_{1-x} \mathrm{Mn}_{x} \mathrm{Sb}$ alloys", Physica E 20, 325 (2004).

162. H. Luo, G. B. Kim, M. Cheon, X. Chen, M. Na, S. Wang, B. D. McCombe, X. Liu, Y. Sasaki, T. Wojtowicz, J. K. Furdyna, G. Boishin, and L. J. Whitman, "Ferromagnetic GaSb/Mn digital alloys", Physica E 20, 338 (2004).

163. W. L. Lim, T. Wojtowicz, X. Liu, M. Dobrowolska, and J. K. Furdyna, "MBE growth and magnetotransport studies of ferromagnetic $\mathrm{Ga}_{1-x} \mathrm{Mn}_{x} \mathrm{Sb}$ semiconductor layers on hybrid GaAs/ZnTe substrates", Physica E 20, 346 (2004).

164. G. B. Kim, M. Cheon, S. Wang, H. Luo, B. D. McCombe, X. Liu, Y. Sasaki, and J. K. Furdyna, "Electric-field control of ferromagnetism in GaSb/Mn digital alloys", Physica E 20, 355 (2004).

165. X. Liu, W. L. Lim, L. V. Titova, T. Wojtowicz, M. Kutrowski, K. J. Yee, M. Dobrowolska, J. K. Furdyna, S. J. Potashnik, M. B. Stone, P. Schiffer, I. Vurgaftman, and J. R. Meyer, "External control of the direction of magnetization in ferromagnetic InMnAs/GaSb heterostructures", Physica E 20, 370 (2004).

166. G. Acbas, G. B. Kim, X. Chen, S. Wang, M. Cheon, C. J. Meining, H. Luo, B. D. McCombe, Y. Sasaki, X. Liu and J. K. Furdyna, "Magnetotransport and magnetic properties of InAs/Mn digital alloys", Physica E 20, 382 (2004).

167. I. Kuryliszyn-Kudelska, J. Z. Domagala, T. Wojtowicz, X. Liu, E. Lusakowska, W. Dobrowolski, and J. K. Furdyna, "The Effect of Mn Interstitials on the Lattice Parameter of $\mathrm{Ga}_{1-x} \mathrm{Mn}_{x} \mathrm{As}$ ", J. Appl. Phys. 95, 603 (2004).

168. I. Kuryliszyn-Kudelska, T. Wojtowicz, X. Liu, J.K. Furdyna, W. Dobrowolski, J.Z. Domagala, E. Łusakowska, M. Goiran, E. Haanappel, and O. Portugall, "Effect of annealing on magnetic and magnetotransport properties of $\mathrm{Ga}_{1-x} \mathrm{Mn}_{x} \mathrm{As}$ epilayers", J. Magnetism and Magnetic Materials 272-276, e1575 (2004).

169. Y. Jiang, R. Pasternak, Z. Marka, Y. V. Shirokaya, J. K. Miller, S. N. Rashkeev, Y. D. Glinka, I. E. Perakis, P. K. Roy, J. Kozub, B. K. Choi, D. M. Fleetwood, R. D. Schrimpf, X. Liu, Y. Sasaki, J. K. Furdyna and N. H. Tolk, "Spin/carrier dynamics at semiconductor interfaces using intense, tunable, ultra-fast lasers", Phys. Status Solidi B 240, 490 (2003).

170. S. U. Yuldashev, H. Im, V. S. Yalishev, C. S. Park, T. W. Kang, S. Lee, Y. Sasaki, X. Liu and J. K. Furdyna, "Magnetoresistance of $\mathrm{Ga}_{1-x} \mathrm{Mn}_{x}$ As epitaxial layers doped by Be", Jpn. J. Appl. Phys. 1 42, 6256 (2003).

171. C. Rüester, T. Borzenko, C. Gould, G. Schmidt, L. W. Molenkamp, X. Liu, T. J. Wojtowicz, J. K. Furdyna, Z. G. Yu, and M. E. Flatte, "Very large magnetoresistance in lateral ferromagnetic (Ga,Mn)As wires with nanoconstrictions", Phys. Rev. Lett. 91, 216602 (2003).

172. T. Wojtowicz, W.L. Lim, X. Liu, M. Dobrowolska, J. K. Furdyna, K. M. Yu, W. Walukiewicz, I. Vurgaftman, and J. R. Meyer, "Enhancement of Curie temperature in $\mathrm{Ga}_{1-x} \mathrm{Mn}_{x} \mathrm{As} / \mathrm{Ga}_{1-y} \mathrm{Al}_{y} \mathrm{As}$ ferromagnetic heterostructures by Be modulation doping", Appl. Phys. Lett. 83, 4220 (2003).

173. F. C. Peiris, B. A. Kowalski, X. Liu, U. Bindley, and J. K. Furdyna, "Optical properties of molecular beam epitaxy-grown $\mathrm{Zn}_{1-x} \mathrm{Mn}_{x} \mathrm{Te}$ thin films measured by complementary techniques", J. Appl. Phys. 94, 4717 (2003).

174. K. M. Yu, W. Walukiewicz, T. Wojtowicz, W. L. Lim, X. Liu, U. Bindley, M. Dobrowolska and J. K. Furdyna, "Curie temperature limit in ferromagnetic $\mathrm{Ga}_{1-x} \mathrm{Mn}_{x} \mathrm{As}$ ", Phys. Rev. B 68, 041308(R) (2003).

175. Y. L. Soo, S. Wang, S. Kim, G. Kim, M. Cheon, X. Chen, H. Luo, Y. H. Kao, Y. Sasaki, X. Liu, and J. K. Furdyna, "Variations of long- and short-range-order structural and magnetic properties of thermally annealed $\mathrm{Mn} / \mathrm{GaAs}$ digital alloys", Appl. Phys. Lett. 83, 2354 (2003).

176. Y. L. Soo, G. Kioseoglou, S. Kim, X. Chen, H. Luo, Y. H. Kao, H.-J. Lin, H. H. Hsieh, T. Y. Hou, C. T. Chen, Y. Sasaki, X. Liu, and J. K. Furdyna "Local environment surrounding ferromagnetically ordered Mn in $\mathrm{Mn} / \mathrm{GaAs}$ digital alloys and (Mn, Ga)As random alloys", Phys. Rev. B 67, 214401 (2003).

177. X. Liu, Y. Sasaki, J. K. Furdyna, "Ferromagnetic resonance in $\mathrm{Ga}_{1-\mathrm{x}} \mathrm{Mn}_{\mathrm{x}}$ As: Effects of magnetic anisotropy", Phys. Rev. B 67, 205204 (2003).

178. T. Wojtowicz, G. Cywiński, W. L. Lim, X. Liu, M. Dobrowolska and J. K. Furdyna, K. M. Yu, W. Walukiewicz, G. B. Kim, M. Cheon, X. Chen, S. M. Wang, and H. Luo, " $\mathrm{In}_{1-x} \mathrm{Mn}_{x} \mathrm{Sb}$-- a narrow-gap ferromagnetic semiconductor", Appl. Phys. Lett. 82, 4310 (2003).

179. S. Lee, S. J. Chung, I. S. Choi, Sh. U. Yuldashev, Hyunsik Im, T. W. Kang, W. L. Lim, Y. Sasaki, X. Liu, T. Wojtowicz, and J. K. Furdyna, "Effect of Be doping on the properties of GaMnAs ferromagnetic semiconductors", J. Appl. Phys. 93, 8307 (2003). 
180. U. Welp, V. K. Vlasko-Vlasov, X. Liu, J. K. Furdyna, and T. Wojtowicz "Magnetic Domain Structure and Magnetic Anisotropy in $\mathrm{Ga}_{1-\mathrm{x}} \mathrm{Mn}_{\mathrm{x}} \mathrm{As}$ ", Phys. Rev. Lett. 90, 167206 (2003).

181. T. Wojtowicz, W. L. Lim, X. Liu, Y. Sasaki, U. Bindley, M. Dobrowolska, J. K. Furdyna, K. M. Yu, and W. Walukiewicz, "Correlation of Mn lattice location, free hole concentration, and Curie temperature in ferromagnetic GaMnAs", Journal of Superconductivity 16, 41 (2003).

182. I. Kuryliszyn, T. Wojtowicz, X. Liu, J. K. Furdyna, W. Dobrowolski, J.-M. Broto, M. Goiran, O. Portugall, H. Rakoto, and B. Raquet,"Low temperature annealing studies of $\mathrm{Ga}_{1-x} \mathrm{Mn}_{x} \mathrm{As}$ ", Journal of Superconductivity 16, 63 (2003).

183. Y. Sasaki, X. Liu, T. Wojtowicz, and J. K. Furdyna, "Spin Wave Resonances in GaMnAs", Journal of Superconductivity 16, 143 (2003).

184. J. K. Furdyna, X. Liu, W. L. Lim, Y. Sasaki, T. Wojtowicz, I. Kuryliszyn, S. Lee, K. M. Yu, and W. Walukiewicz, "Ferromagnetic III-Mn-V Semiconductors: Manipulation of Magnetic Properties by Annealing, Extrinsic Doping, and Multilayer Design", Journal of the Korean Physical Society 42, S579-S590 (2003).

185. Yu. D. Glinka, T. V. Shahbazyan, I. E. Perakis, N. H. Tolk, X. Liu, Y. Sasaki, and J. K. Furdyna, "Pump-probe second harmonic generation study of ultrafast spin dynamics in semiconductor multilayers", Surface and Interface Analysis. 35, 146 (2003).

186. Sh. U. Yuldashev, Hyunsik Im, V. Sh. Yalishev, C. S. Park, T. W. Kang, Sanghoon Lee, Y. Sasaki, X. Liu, and J. K. Furdyna, "Effect of additional nonmagnetic acceptor doping on the resistivity peak and the Curie temperature of $\mathrm{Ga}_{1-x} \mathrm{Mn}_{x}$ As epitaxial layers", Appl. Phys. Lett. 82, 1206 (2003)

187. B. D. McCombe, M. Na, X. Chen, M. Cheon, S. Wang, H. Luo, X. Liu, Y. Sasaki, T. Wojtowicz, J. K. Furdyna S. J. Potashnik and P. Schiffer, "Novel ferromagnetism in digital GaAs/Mn and GaSb/Mn alloys", Physica E 16, 90 (2003).

188. Yu. D. Glinka, T. V. Shahbazyan, I. E. Perakis, N. H. Tolk, X. Liu, Y. Sasaki, and J. K. Furdyna, "Ultrafast dynamics of interfacial electric fields in semiconductor heterostructures monitored by pump-probe secondharmonic generation", Appl. Phys. Lett. 81, 3717 (2002).

189. I. Kuryliszyn, T. Wojtowicz, X. Liu, J. K. Furdyna, W. Dobrowolski, J.-M. Broto, M. Goiran, O. Portugall, H. Rakoto, and B. Raquet, "Transport and magnetic properties of low temperature annealed $\mathrm{Ga}_{1-\mathrm{x}} \mathrm{Mn}_{\mathrm{x}} \mathrm{As}$ ", Acta Physica Polonica A. 102, 659 (2002).

190. X. Liu, Y. Sasaki, L. V. Titova, P. M. Reimer, S. Lee and J. K. Furdyna, "Fabrication and characterization of III-V semiconductor superlattices with sinusoidal compositional modulation", Physica E 13, 1143 (2002).

191. D. V. Baxter, D. Ruzmetov, J. Scherschligt, Y. Sasaki, X. Liu, J. K. Furdyna, and C. H. Mielke, "Anisotropic magnetoresistance in $\mathrm{Ga}_{1-x} \mathrm{Mn}_{x} \mathrm{As}$ ”, Phys. Rev. B 65, 212407 (2002).

192. K. M. Yu, W. Walukiewicz, T. Wojtowicz, W. L. Lim, X. Liu, Y. Sasaki, M. Dobrowolska, and J. K. Furdyna, "Determination of free hole concentration in ferromagnetic $\mathrm{Ga}_{1-x} \mathrm{Mn}_{x} \mathrm{As}$ using electrochemical capacitancevoltage profiling", Appl. Phys. Lett. 81, 844-846 (2002).

193. Yu. D. Glinka, T. V. Shahbazyan, I. E. Perakis, N. H. Tolk, X. Liu, Y. Sasaki, and J. K. Furdyna, "Ultrafast spin dynamics in GaAs/GaSb/InAs heterostructures probed by second harmonic generation", Appl. Phys. Lett. 81, 220 (2002).

194. X. Chen, M. Na, M. Cheon, S. Wang, H. Luo, B. D. McCombe, X. Liu, Y. Sasaki, T. Wojtowicz, J. K. Furdyna, S. J. Potashnik and P. Schiffer, "Above-room-temperature ferromagnetism in GaSb/Mn digital alloys", Appl. Phys. Lett. 81, 511-513 (2002).

195. K. M. Yu, W. Walukiewicz, T. Wojtowicz, I. Kuryliszyn, X. Liu, Y. Sasaki, and J. K. Furdyna, "Effect of the location of $\mathrm{Mn}$ sites in ferromagnetic $\mathrm{Ga}_{1-x} \mathrm{Mn}_{x} \mathrm{As}$ on its Curie temperature", Phys. Rev. B 65, 201303(R) (2002).

196. Y. Sasaki, X. Liu, J. K. Furdyna, M. Palczewska, J. Szczytko, and A. Twardowski, "Ferromagnetic resonance in GaMnAs", J. Appl. Phys. 91, 7484 (2002).

197. J. K. Furdyna, X. Liu, Y. Sasaki, S. J. Potashnik, and P. Schiffer, "Ferromagnetic III-Mn-V semiconductor multilayers: Manipulation of magnetic properties by proximity effects and interface design (invited)", J. Appl. Phys. 91, 7490 (2002).

198. H. Luo, B. D. McCombe, M. H. Na, K. Mooney, F. Lehmann, X. Chen, M. Cheon, S. M. Wang, Y. Sasaki, X. Liu and J. K. Furdyna, "Transport and magnetic properties of ferromagnetic GaAs/Mn digital alloys", Physica E 12, 366 (2002).

199. Y. L. Soo, G. Kioseoglou, S. Kim, X. Chen, H. Luo, Y. H. Kao, Y. Sasaki, X. Liu, and J. K. Furdyna, "Studies of $\mathrm{Mn} / \mathrm{GaAs}$ digital alloys using $\mathrm{x}$-ray absorption fine structure and x-ray diffraction methods", Appl. Phys. Lett. 80, 2654 (2002). 
200. X. Liu, U. Bindley, Y. Sasaki, and J. K. Furdyna, "Optical properties of epitaxial ZnMnTe and ZnMgTe films for a wide range of alloy compositions", J. Appl. Phys. 91, 2859 (2002).

201. G. Kioseoglou, S. Kim, Y. L. Soo, X. Chen, H. Luo, Y. H. Kao, Y. Sasaki, X. Liu, and J. K. Furdyna, "Investigation of nanoscale structure in digital layers of $\mathrm{Mn} / \mathrm{GaAs}$ and $\mathrm{MnGa} / \mathrm{GaAs}$ ", Appl. Phys. Lett. 80, 1150 (2002).

202. X. Liu, Y. Sasaki, and J. K. Furdyna, "Enhancement of magnetic coercivity and ferromagnetic transition temperature by proximity effects in the GaMnAs-ZnMnSe multilayer system", Appl. Phys. Lett. 79, 2414 (2001).

203. Youngho Um, S. Lee, X. Liu, and J. K. Furdyna, "Enhancement of the type-I transition in type-II ZnTe/CdSe Bragg confining structures", Journal-of-the-Korean-Physical-Society. 39, 429-32 (2001).

204. Youngho Um, S. Lee, X. Liu, and J. K. Furdyna, "Enhancement of direct (type-I) excitonic transitions in ZnTe/CdSe-based type-II Bragg confining structures", J. Appl. Phys. 89, 5460 (2001).

205. S. Lee, G. Yang, X. Liu, U. Bindley, M. Dobrowolska, J. K. Furdyna, P. M. Reimer and John R. Buschert, "Fabrication and optical properties of $\mathrm{ZnSeTe}$ superlattices with sinusoidal compositional modulation", J. Crystal Growth, 214-215, 25 (2000).

Book Chapters

206. J. K. Furdyna, S. Lee, M. Dobrowolska, T. Wojtowicz, and X. Liu, "Band-Offset Engineering in Magnetic/Non-Magnetic Semiconductor Quantum Structures", in Introduction to the Physics of Diluted Magnetic Semiconductors, Springer Series in Materials Science, Vol. 144, edited by Jacek Kossut and Jan A. Gaj (Springer-Verlag, Berlin2010).

207. K. M. Yu, T. Wojtowicz, W. Walukiewicz, X. Liu, J. K. Furdyna, "Fermi Level Effects on Mn Incorporation in III-Mn-V Ferromagnetic Semiconductors", in Spintronics, Semiconductors and Semimetals, Vol. 82, edited by Tomasz Dietl, David D. Awachalom, Maria kamińska, Hideo Ohno (Elsevier, San Diego, 2008).

208. J. K. Furdyna, X. Liu, T. Wojtowicz, W. L. Lim, U. Welp, V. K. Vlasko-Vlasov, "Magnetic Anisotropy in Ferromagnetic III-Mn-V Semiconductors: Issues and Observations", in Advances in Solid State Physics, Vol. 44, edited by B. Kramer (Springer, Berlin, 2004).

209. J. K. Furdyna, P. Schiffer, Y. Sasaki, S. J. Potashnik, and X. Y. Liu, "Ferromagnetic semiconductors and their nanostructures: new opportunities and challenges", in Optical Properties of Semiconductor Nanostructures, NATO Science Series, Vol. 81, edited by M. L. Sadowski, M. Potemski, and M. Grynberg (Kluwer, Dordrecht, 2000).

Conference Proceedings

210. "Four stable magnetization states formed in a single layer of GaMnAs ferromagnetic film", S. Lee, X. Liu, and J. K. Furdyna, Materials Research Society Symposium Proceedings 1183, 37-42 (2010).

211. "Spin dynamics and manipulation in GaMnAs alloys", X. Liu, Y. Y. Zhou, E. Harley, L. E. McNeil, J. Wang, J. Qi, Y. Xu, A. Steigerwald, N. Tolk, J. P. Zahn, A. Gamouras, S. March, K. C. Hall, J. K. Furdyna, Proc. SPIE 7600, 76000T (2010).

212. “CdSe/ZnTe heterojunction solar cells grown on GaSb”, S. Wang, D. Ding, R. Scott, J. Chen, M. DiNezza, X. Liu, J. K. Furdyna, Y.-H. Zhang, Proc. 34rd IEEE Photovoltaic Specialists Conference, 112, (2009).

213. "Four stable magnetization states formed in a single layer of GaMnAs ferromagnetic film", Sanghoon Lee, X. Liu, J. K. Furdyna, Materials Research Society Symposium Proceedings. Novel Materials and Devices for Spintronics (2009).

214. "Memory effects in femtosecond collective spin rotation in ferromagnetic semiconductors", J. Wang, I. Cotoros, D. S. Chemla, X. Liu, J. K. Furdyna, J. Chovan, and I. E. Perakis, Proc. SPIE 7214, 721412 (2009).

215. "Lattice-matched ZnTe and CdZnTe/ZnTe heterostructures grown on GaSb for multijunction solar cell applications", S. Wang, X. Liu, D. Ding, S.-N. Wu, S. R. Johnson, S.-Q. Yu, J. K. Furdyna, Y.-H. Zhang, Proc. 33rd IEEE Photovoltaic Specialists Conference, 1465, (2008).

216. "Ultrafast Coercivity and Magnetization Dynamics in GaMnAs", K. C. Hall, J. P. Zahn, S. March, X. Liu, J. K. Furdyna, Proc. 2008 Conference on Lasers and Electro-Optics and Quantum Electronics and Laser Science (CLEO/QELS), 3329 (2008).

217. "Ultrafast photo-enhanced ferromagnetism in GaMnAs", Jigang Wang, Ingrid Cotoros, Xinyu Liu, Jacek K. Furdyna, and Daniel S. Chemla, Proc. SPIE 6892, 68920Q (2008).

218. "Control of coherent magnetization precession in GaMnAs by ultrafast optical excitation”, J. Qi, Y. Xu, X. Liu, J. K. Furdyna, I. E. Perakis, and N. Tolk, Proc. SPIE 6839, 68390J (2007). 
219. "Kerr Rotation and Magnetic Circular Dichroism in Ferromagnetic InMnSb and InMnAs", A. Winter, H. Pascher, H. Krenn, T. Wojtowicz, X. Liu, and J. K. Furdyna, AIP Conf. Proc. 893, 1223 (2007). Proc. 28th Int. Conf. on Physics of Semiconductors.

220. "Magnetic cluster phases of Mn-interstitial-free ( $G a, M n) A s$ ", Y. J. Cho, M. A. Scarpulla, X. Liu, Y. Y. Zhou, O. D. Dubon, and J. K. Furdyna, AIP Conf. Proc. 893, 1221 (2007). Proc. 28th Int. Conf. on Physics of Semiconductors.

221. "Ferromagnetic Resonance Study of Ultra-thin $G a_{1-x} M n_{x} A s$ Films as a Function of Layer Thickness", Y. Y. Zhou, Y. J. Cho, Z. Ge, X. Liu, M. Dobrowolska, and J. K. Furdyna, AIP Conf. Proc. 893, 1213 (2007). Proc. 28th Int. Conf. on Physics of Semiconductors.

222. "Carrier Dynamics and Magnetization-induced Nonlinearity in Ferromagnetic GaMnAs", Ji-Hee Kim, KangJeon Han, Ki-Ju Yee, X. Liu, J. K. Furdyna, and Y. S. Lim, AIP Conf. Proc. 893, 1207 (2007). Proc. 28th Int. Conf. on Physics of Semiconductors.

223. "Ultrafast Magneto-Optical Kerr Study of Standing Spin Waves in Ferromagnetic GaMnAs Films", D. M. Wang, Y. H. Ren, X. Liu, Y. J. Cho, J. K. Furdyna, M. Grimsditch, and R. Merlin, AIP Conf. Proc. 893, 1175 (2007). Proc. 28th Int. Conf. on Physics of Semiconductors.

224. "Magnetic anisotropy of strain-engineered InMnAs ferromagnetic films and easy-axis manipulation from outof-plane to in-plane orientations", X. Liu, W. L. Lim, Z. Ge, S. Shen, T. Wojtowicz, K. M. Yu, W. Walukiewicz, M. Dobrowolska, and J. K. Furdyna, AIP Conf. Proc. 772, 367 (2005), Proc. 27th Int. Conf. on Physics of Semiconductors.

225. "Optical investigation of temperature-induced changes in magnetic anisotropy in III-Mn-As ferromagnetic semiconductors”, M. Kutrowski, L. Titova, R. Chakarvorty, K. Yee, W. L. Lim, X. Liu, T. Wojtowicz, J. K. Furdyna, and M. Dobrowolska, AIP Conf. Proc. 772, 361 (2005), Proc. 27th Int. Conf. on Physics of Semiconductors.

226. "Electrical, Magnetic and Magnetooptical Properties of Bulk (Zn,Mn)Te Semimagnetic Semiconductor Doped with Phosphorus", Van Khoi Le, R. R. Galazka, M. Dobrowolska, K. Yee, X. Liu, W.-L. Lin, J. K. Furdyna, and T. M. Giebultowicz, AIP Conf. Proc. 772, 337 (2005), Proc. 27th Int. Conf. on Physics of Semiconductors.

227. "Effects of Mn Site Location on the Magnetic Properties of $I I I_{-x} M n_{x} V$ Semiconductors", K. M. Yu, W. Walukiewicz, T. Wojtowicz, J. Denlinger, X. Liu, and J. K. Furdyna, AIP Conf. Proc. 772, 303 (2005), Proc. 27th Int. Conf. on Physics of Semiconductors - invited.

228. "Magnetic circular dichroism in $\mathrm{ZnSe} / \mathrm{Ga}_{1-x} \mathrm{Mn}_{x} \mathrm{As}$ hybrid structures with Be and Si co-doping", R. Chakarvorty, K. J. Yee, X. Liu, P. Redlinski, M. Kutrowski, L. V. Titova, T. Wojtowicz, J. K. Furdyna, B. Janko, and M. Dobrowolska, AIP Conf. Proc. 772, 1337 (2005), Proc. 27th Int. Conf. on Physics of Semiconductors.

\section{PROFESSIONAL REFERENCES}

Professor Jacek K. Furdyna

Department of Physics, University of Notre Dame

309 Nieuwland Science Hall, Notre Dame, IN 46556

Email: Jacek.K.Furdyna.1@nd.edu

Professor Malgorzata Dobrowolska

Department of Physics, University of Notre Dame

333B Nieuwland Science Hall, Notre Dame, IN 46556

Email: mdobrowo@nd.edu

Dr. James J. Rhyne

Deputy Group Leader for Science

Lujan Neutron Scattering Center, MS H805

Los Alamos National Laboratory, Los Alamos, NM 87545

Email: rhyne@lanl.gov

Professor Hong Luo

Department of Physics, University at Buffalo, The State University of New York

126 Fronczak Hall, North Campus, Buffalo, NY 14260

Email: luo@buffalo.edu

Dr. Ulrich Welp 
Superconductivity and Magnetism Group, Argonne National Laboratory

9700 S. Cass Ave., Argonne, IL 60439

Email: welp@anl.gov

Dr. Wladyslaw Walukiewicz

Materials Sciences Division, Ernest Orlando Lawrence Berkeley National Laboratory

One Cyclotron Road, Berkeley, CA 94720

Email:w_walukiewicz@lbl.gov

Professor Tomasz Wojtowicz

Institute of Physics, Polish Academy of Sciences

Al. Lotnikow 32/46, Warsaw, 02-681, POLAND

Email: wojto@ifpan.edu.pl

Prof. Yong-Hang Zhang

Department of Electrical Engineering, Arizona State University

ERC 161, Tempe, AZ 85287-5706

Email: yhzhang@asu.edu

Date: 28-Apr-11 